\title{
LA CONTRIBUTIO ENTRE ILICI E ICOSIUM: UN CANDENTE DEBATE HISTORIOGRÁFICO
}

THE CONTRIBUTIO BETWEEN ILICI AND ICOSIUM: A BURNING HISTORIOGRAPHICAL DEBATE

JUAN JOSÉ SEGUÍ MARCO

Universitat de València

Juan.j.segui@uv.es

http://orcid.org/0000-0002-4787-5061

Recepción: 31-05-2017

Aceptación: 08-08-2017

\section{Resumen}

La contributio entre Ilici e Icosium, sólo conocida por un texto de Plinio, se revela como uno de los debates historiográficos internacionales más interesantes de los últimos años, con posturas muy dispares y múltiples matices. Hemos recogido de forma cronológica el conjunto de opiniones que a lo largo del tiempo han marcado los diversos puntos de vista, desde los orígenes de la polémica hasta la actualidad.

Palabras clave. Contributio; Ilici; Icosium.

\begin{abstract}
The contributio between Ilici and Icosium, only known of thanks to a text by Pliny, has become one of the most interesting international historiographical debates in the last few years, with very different postures and multiple nuances. We have collected chronologically the set of opinions that have marked over time the various points of view from the beginning of the controversy to the present.
\end{abstract}

Key words. Contributio; Ilici; Icosium. 


\section{LOS PRIMEROS AÑOS: UNA POLÉMICA DE BAJA INTENSIDAD}

Por un texto de la Historia Naturalis de Plinio dedicado a las ciudades de la costa de Hispania Citerior (Fig. 1), sabemos que había una relación contributense entre Ilici e Icosium. ${ }^{1}$

Las referencias más antiguas que recogen esta circunstancia las podemos rastrear en los mismos inicios de la historiografía moderna, partiendo de los cronistas valencianos del siglo XVI hasta llegar a los historiadores pre-positivistas. Situada en esa última frontera, en la obra clásica sobre Elche en la Antigüedad de A. Ibarra (1879: 216-219), podemos consultar una detallada referencia de todas estas menciones (Beuter, Diago, Strany, Mayans, Boix), marcadas siempre por la obsesión de encontrar la ubicación de Icosium, algo que para este autor no era posible, por entender que más que habitantes de una ciudad eran residentes de una cercana comarca. A las reseñas que aporta este gran erudito decimonónico sólo debemos añadir una que pasa por alto, y que, aunque sólo sea por su reutilización por la historiografía posterior -omitiendo siempre su autoría original-, merece ser recordada. Nos referimos a la propuesta de M. Cortés y López (1836: 62-63), según la cual, los icositanos se encontraban en Agost, pues el nombre moderno había mudado el antiguo por cambio de la I por la A y la C por G.

No obstante, esta perspectiva localista que hasta este momento había ofrecido el tema, estaba pronta a cambiar ante los avances que estaba realizando la historiografía europea, especialmente la alemana, encaminada a recoger exhaustivamente toda la información que procediera del mundo antiguo. La irrupción de la contributio icositana a escala internacional hubo de esperar, sin embargo, hasta 1918, cuando fue incorporada en la publicación del tercer Supplementum de la $R E$ con una referencia de J. B. Keune (1918: 1195), donde se establecía un nexo entre los icositani mencionados por Plinio y la ciudad de Icosium en el norte de África. Parece bastante claro que la historiografía alemana intentaba reparar su inexplicable omisión del tema en los comentarios del CIL VIII para el África romana de G. Wilmanns (1881: 794).

Como era de esperar, muy pronto se le sumarían las reseñas de la historiografía francesa. En efecto, S. Gsell (1921-1928: 204), en su insigne obra sobre la historia antigua del norte de África, reconocía también que el Icosium pliniano era la ciudad norteafricana y sugería que la colonia ilicitana hubiera podido acoger en época augústea a ciudadanos romanos procedentes de ella. El investigador francés añadía, además, que con el restablecimiento del reino de Mauritania con Juba II, las otras colonias fundadas por Augusto en la zona mantendrían, como Zulil e Icosium, la dependencia de Hispania.

No mucho después, E. Albertini (1923: 41, n. 2), al estudiar la división administrativa realizada por el primer emperador romano en la península ibérica, mantenía un punto de vista similar al opinar que estas dos ciudades, unidas respectivamente a la Bética y a la Tarraconense, se mostraban como un ejemplo de lo que debió ocurrir con las otras colonias romanas presentes en el reino mauritano de Juba II. Los icositani eran, para este investigador, sin ninguna duda, los habitantes del moderno Argel, y, por tanto, no había que buscarlos en España, pues «Pline n'aurait aucune raison d'accorder une mention à ce locus contributus, seul entre tous ceux de la Citèrieur» (Albertini, 1923: 6566). Añadía, además, una propuesta cronológica, pues

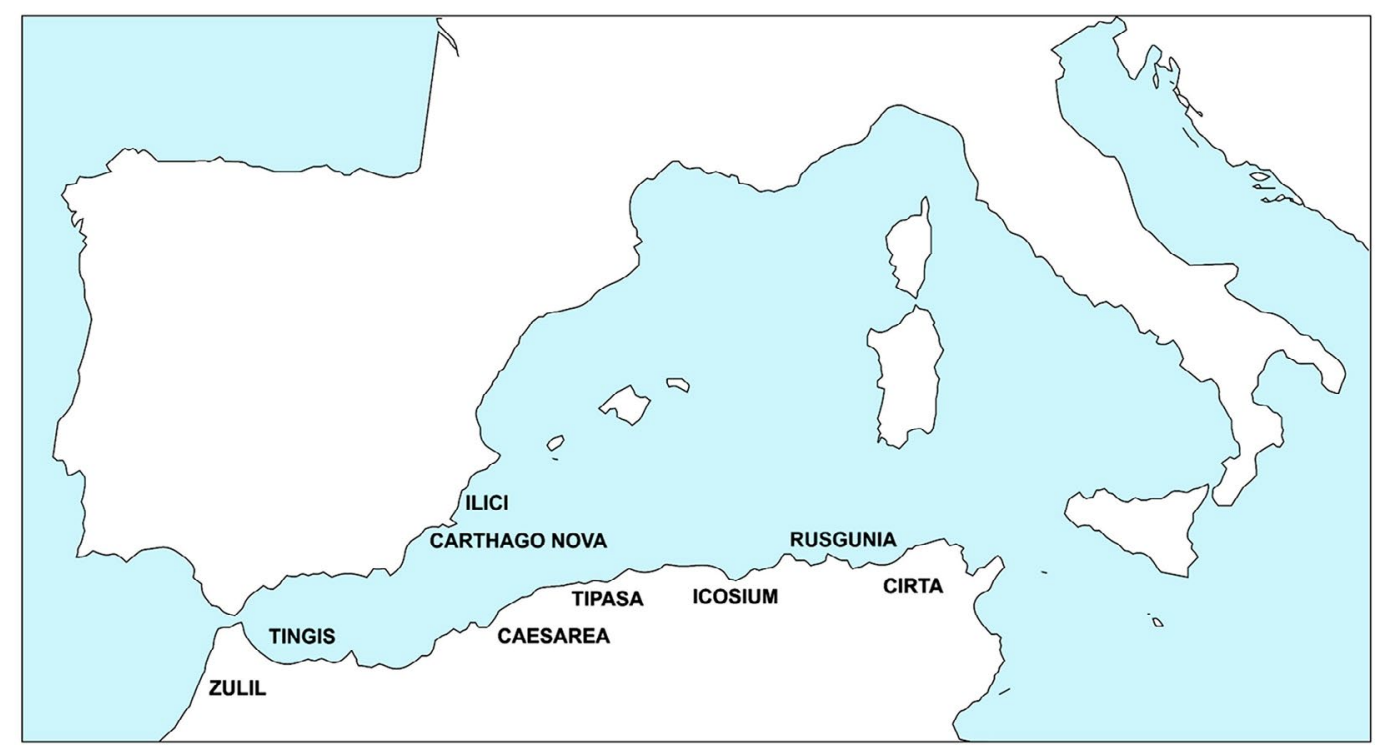

Figura 1: Mapa de las principales localidades citadas en el texto. Elaboración del autor.

1. Plin. III 4, 19: Reliqua in ora flumen Tader, colonia inmunis

Ilici, unde Ilicitanus sinus: in eam contribuuntur Icositani. 
opinaba que la extinción del vínculo contributense se habría consumado en el reinado de Claudio, coincidiendo con la creación de la provincia de Mauritania.

Estas iban a ser las pautas dentro de las cuales se movería, grosso modo, la investigación durante los años sucesivos, en una alternancia entre las opiniones de la historiografía francesa y la alemana que iba a ser la tónica constante de ahora en adelante. Y así, E. Kornemann, (1940: 91-92), representante de esta última, daba por sentado que nos encontrábamos ante la ciudad mauritana de Icosium y, ya que le resultaba inconcebible una relación entre una ciudad privilegiada y una comunidad peregrina, proponía la existencia previa de una pequeña colonia de ciudadanos - «kleine Bürgerkolonie»)- y que sólo con ella se habría establecido la contributio, dejando fuera de la misma a los indígenas o peregrinos. El investigador alemán entendía que la extinción del vínculo entre Ilici e Icosium y la plena integración de la comunidad indígena eran tardías, esto es, entre los años 74-76 d.C., cuando la localidad recibiría el título de colonia de derecho latino, según constaba en otro conocido texto pliniano. ${ }^{2}$ E. Kornemann (1940: 92) planteaba que, por razones geográficas, la contributio de la colonia de Ilici se ejercería sobre Icosium mediante unos mecanismos similares a los que empleó Cirta sobre su confederación, esto es, haciendo uso de unos praefecti i(ure) d(icundo).

De aquí en adelante, la propuesta de una reducida colonia de ciudadanos romanos fundada por Augusto en Icosium, germen de la contributio, fue acogida de muy diversa manera por la historiografía alemana. Así, mientras F. Vittinghoff (1951: 117, n. 4) se opuso rotundamente a su existencia, L. Teutsch (1962: 200201), un decenio después, en el marco de un estudio general sobre la evolución de la presencia romana en el norte de África, en el que se destacaba el valor de los conventus civium Romanorum en el proceso de urbanización del territorio, insistía en que Icosium debió contar bajo Augusto, al menos, con uno de estos conventus, desde el cual se establecería la contributio con Ilici. L. Teutsch (1962: 201, n. 543) recogía y reelaboraba, ahora sí de forma explícita, las anteriores sugerencias de S. Gsell y de E. Kornemann. En tal sentido, opinaba que las provincias hispanas habrían actuado como supervisoras de las colonias norteafricanas cuando Mauritania quedó bajo la autoridad de Juba II, al igual que habría ocurrido con Icosium y Zulil (Teusch, 1962: 220-221). Este vínculo entre Ilici e Icosium se habría mantenido hasta comienzos del gobierno de Vespasiano -siguiendo así la opinión de E. Kornemann- hasta interrumpirse en el momento en

2. Plin. V, 20: Promontorium Apollinis oppidumque ibi celeberrimum Caesarea, ante vocatum Iol, Iubae regia a Divo Claudio coloniae iure donata, eiusdem iussu deductis veteranis Oppidum Novum at Latio dato Tipasa, itemque a Vespasiano Imperatore eodem munere donatum Icosium. que los icositanos recibieron el derecho latino, según el mencionado texto de Plinio (Teusch, 1962: 201, n. 544).

\section{HACIA UNA MEJOR DEFINICIÓN DEL PROBLEMA}

La publicación por U. Laffi (1966: 13-17 y 163) de una extensa monografía sobre la cuestión de la contributio romana, marcó un momento clave en la definición teórica y práctica de esta institución. Después de analizar las propuestas anteriores, consideraba que nos hallábamos ante un régimen administrativo romano asociado al derecho público, que ligaba a dos comunidades urbanas en una relación de estrecha dependencia. La contributio establecía un principio de excepción a la plena autonomía urbana que presidía el derecho local romano, al permitir una integración asimétrica entre ciudades que, en palabras de U. Laffi (1966: 159), se caracterizaba por «la fusione di due o piú comunità autonome in un'unica comunità, che assommava e accentrava anche le funzioni amministrative e giurisdizionali delle comunità contributa. Tale definicione dovrà tuttavia essere precisata nel senso che le comunità interessate dal provvedimento erano, di regola, comunità cittadine.» Así pues, los contributenses serían, en su mayoría, ya ciudadanos romanos antes de establecerse este régimen entre dos ciudades, cuya jurisdicción la ostentarían los magistrados de la ciudad dominante. A cambio, los cives de la comunidad contributense podrían presentarse a las elecciones de magistrados de la ciudad rectora. Por tanto, existía una paridad de derechos entre unos y otros, si bien era posible que la ciudad subordinada conservara una cierta autonomía en la administración ordinaria, postura en la que U. Laffi (1966: 161-162 y n. 501) difería de la opinión de E. Korneman. En cuanto a los motivos de la constitución de este tipo de vínculos, el investigador italiano los veía tanto como un medio para organizar administrativa y financieramente dos comunidades sitas en un área concreta, como un principio de racionalización económica, pues al asociarse, la que se encontraba en una situación de inferioridad podía obtener un alivio en los gastos que comportaba una administración propia, al tiempo que también favorecía con su apoyo a la comunidad dirigente. Desde luego, U. Laffi (1966: 163-164) entendía la vigencia de esta institución como transitoria, pues con el tiempo cada ciudad adquiriría plena independencia. Para el caso que nos ocupa, el de Ilici-Icosium, opinaba que habría sido una consecuencia del proceso de colonización de veteranos tras la batalla de Actium, entre el 33 y el 25 a.C., momento en que Mauritania dependía de Roma. A partir de esa fecha, todas las comunidades ciudadanas fundadas por Augusto habrían pasado a la jurisdicción de las provincias hispanas. Plinio, opinaba U. Laffi (1966: 120122 y n. 504), recogía la contributio porque figuraba en la formula provinciae Tarraconensis, anterior a la 
constitución de las provincias mauritanas, y consideraba que la aplicación de este régimen administrativo entre las ciudades de Ilici e Icosium se llevó a cabo a iniciativa e interés de la autoridad romana, adscribiendo a los icositanos a dos jurisdicciones, una menor, con la colonia hispana, y otra mayor, la del gobernador de la provincia Tarraconense. U. Laffi concebía la relación contributense solamente con el conventus civium Romanorum de Icosium. La situación de contributio finalizaría con la creación de las provincias mauritanas por Claudio, mientras que en época flavia la ciudad norteafricana se transformaría en un centro autónomo de derecho latino. El investigador italiano dejaba sin abordar la situación de la contributio entre la época claudiana y la de Vespasiano.

En el marco de la creciente atención que esta cuestión estaba suscitando se planteó, muy poco después, un nuevo acercamiento, en un estudio de conjunto de la historia de la ciudad icositana que emprendió $\mathrm{M}$. Le Glay (1968: 17). El investigador francés, que consideraba la contributio con Ilici un hecho «curieux», estaba convencido, en consonancia con toda la investigación, de que la ciudad norteafricana era la que Plinio vinculaba con la colonia hispana, aunque no albergaba plena seguridad de que los colonos, a su modo de ver, veteranos romanos poco numerosos, llegaran a formar un conventus civium Romanorum. En su opinión, la inscripción encontrada en Argel y dedicada por $L$. Caecilius Rufus al rey Ptolomeo ${ }^{3}$ era una importante prueba de que, incluso antes de la provincialización, Icosium había contado con magistrados municipales, que mantuvieron excelentes relaciones con el rey mauritano. Para M. Le Glay (1968: 18) esta contributio era el primer testimonio constatado de estrechas relaciones entre Mauritania y la península ibérica.

A finales de la misma década, un singular y documentadísimo libro de J.-M. Lassère (1977: 255-256) sobre las poblaciones indígenas norteafricanas y la romanización, recogía una interesante alusión a la promoción final de Icosium, al considerar que los nombres que aparecen en la epigrafía icositana más antigua (Calpurnius, Ennius, Fadius, Iulius, Sittius) serían de tal momento, destacando el caso de un tal Calpurnius Martialis, Imilis f(ilius), un mauro romanizado, probatorio de los primeros contactos entre romanos e indígenas realizado, según su opinión, sobre un soporte material inspirado en los epitafios cartagineses de época cesariana. Para M. Lassère, la decisión de Vespasiano de desvincular ambas ciudades y transformar Icosium en una colonia se explicaría por la gran expansión de la cultura romana entre la población autóctona.

Llama la atención que la historiografía española, a estas alturas del debate y cuando la contributio entre

3. CIL VIII, 9257: [R]egi Ptolome[o] / reg(is) Iubae f(ilio) / L(ucius) Caecilius Rufus / Agilis f(ilius) honoribus / omnibus patriae / suae consummatis / d(e) s(ua) p(ecunia) $f$ (aciendum) c(uravit) et consacravit.
Ilici e Icosium había despertado el interés internacional desde hacía tiempo, se mantuviera por completo al margen. Si, por un lado, se limitaba a recoger, sin más detalles, esa relación entre la ciudad hispana y la norteafricana, ajena a ninguna polémica (Blázquez, 1978: 652; Tovar, 1989: 198; Ramos, 1975: 160), por otro, todavía mantenía, de forma aislada, la inercia de las tesis que desde principios del s. XVI defendían, según vimos, que Icosium se hallaba en el entorno ilicitano. Fiel a esta concepción, E. Llobregat (1972a: 11; 1972b: 283; 1980: 101) aún sostenía, sin indicar sus fuentes, que se encontraba en la localidad alicantina de Agost, donde identificaba, a través de restos cerámicos, la existencia de una ciudad ibero-romana.

\section{REVISIONES Y ALTERNATIVAS}

A principios de los años ochenta del pasado siglo se llevaron a cabo dos grandes replanteamientos del problema que van a trasladar la cuestión a la primera línea al hilo de los debates sobre el África romana. El primero, cronológicamente, fue el de J. Desanges (1980: 166-169), que en su detallado comentario del libro V de la Historia Naturalis de Plinio, consideraba que la contributio icositana sólo se podía justificar en un momento en que Mauritania estaba, como $Z u$ lil, «regum dicioni exempta»y, puesto que la formula que utiliza Plinio correspondía a época de Augusto, la ciudad habría quedado en aquel momento sustraída a la autoridad de Juba II. La inscripción dedicada por un icositano al rey Ptolomeo no habría que interpretarla como una prueba de dependencia de la ciudad con relación al reino mauritano, pues se trataría simplemente de vínculos personales entre el dedicante $\mathrm{y}$ el monarca, y no de relaciones institucionales. Para J. Desanges (1980: 168), la existencia de un conventus no resultaba convincente al tratarse de asociaciones privadas que no participaban en la gestión de la comunidad peregrina, por lo que sugería que Icosium gozara de un estatuto privilegiado, independiente de la autoridad real, si bien mostraba sus reparos a que un municipio, latino o romano, fuera adscrito a la jurisdicción de una colonia. En cuanto a la finalización de la contributio, J. Desanges (1980: 168) proponía, por primera vez en torno a esta cuestión, un conjunto de reflexiones de gran calado. Puesto que el texto de Plinio sobre África, a datar en época flavia, recogía que Vespasiano acordaba para Icosium ...eodem munere que Tipasa, esto es, el estatuto latino, y una inscripción contemporánea mostraba, entre el 74-76 d.C., a un dunviro quinquenal como el primer pontífice in colonia ${ }^{4}$ habría que concluir -algo aceptado por toda la investigación- que la ciudad pasó entonces a

4. CIL VIII 20853: ... Flaviu[us...]ni/[n]us aed(ilis) (duum) vir [qui]nq/[u]enna(lis) pontife[x p]ri/mus in colonia ex / [d(ecreto)] d(ecurionum) ... 
transformarse en colonia latina. Sin embargo, le resultaba extraño que en época tan tardía se creara este tipo de colonias, desaparecidas en tiempos de Augusto y nunca presentes en África, ni tampoco le parecían admisibles dos promociones sucesivas, una primera a municipio y otra, después, a colonia. Como alternativa proponía que la inscripción del primer pontifex colonial no fuera icositana, sino de la vecina Rusguniae, y que, por tanto, Icosium sólo fuera un municipio desde Vespasiano. No obstante, para J. Desanges (1980: 54 y 168) cabía la alternativa de leer el texto de Plinio V 20 de forma diferente, de manera que Icosium no hubiera recibido del emperador flavio el mismo estatuto que Tipasa, el ius Latii -dato que unido a la referida inscripción conduciría al estatuto colonial latino-, sino un derecho similar al que diera Claudio a Caesarea, por lo que Icosium se habría transformado con Vespasiano en colonia romana. Aunque J. Desanges (1980: 168-169) presentaba precedentes de tal lectura, reconocía que su traducción del texto pliniano se producía en «détriment de la clarité». Además, exigiría que antes Icosium hubiera sido un municipio, pues pensaba que -a diferencia de Caesarea, una antigua capital-, habría necesitado de este paso previo para asegurarse el rango colonial. Como veremos después, toda la historiografía francesa se fue, con el paso del tiempo, alineando con esta propuesta.

El trabajo de J. Desanges se simultaneó con un primer estudio de J. Gascou (1981: 232-233 y 235), en el que respaldaba la hipótesis tradicional de la existencia, en tiempos augústeos, de un conventus civium Romanorum en Icosium, y que fue seguido, al poco, de otro trabajo en el que abordaba directamente la cuestión de la contributio en el marco de los cambios de la política municipal durante los flavios en África del Norte. En el mismo, J. Gascou (1982: 160-161) apoyaba, de nuevo, la existencia de un conventus entre el 33 y el 25 a.C., asociado a Ilici en época de la reinstauración monárquica de Juba II, al tratarse de un régimen propio de ciudadanos romanos con idéntico estatuto personal, y que la vinculación a la ciudad hispana se habría mantenido hasta la anexión de Mauritania como provincia. Después, a partir del 40 d.C., se mantendría el conventus, excluyendo también a los indígenas, aunque algunos de estos, como el individuo que realizó la inscripción al rey Ptolomeo, hijo de un peregrino, ya daban pruebas de que su comunidad se había incorporado al proceso de romanización. Vespasiano, al desear integrar a los ciudadanos romanos del conventus con la población autóctona, se decidió por la fórmula de la colonia latina, pues así satisfacía a los antiguos ciudadanos con un título más honorable que el de municipio, mientras que, al reducirlo a la latinidad, restringía el acceso inmediato de los peregrinos a la ciudadanía romana. Una prueba de esto último la encontraríamos en el pontife $[x$ p]rimus in colonia, un antiguo indígena, promovido a la ciudadanía per honorem como indicaría su gentilicio Flavius. Para J. Gascou (1982: 161 , n. $112^{\mathrm{a}}$ ), la propuesta de J. Desanges, tanto de un municipio previo - pese a que serviría para cubrir el periodo entre Claudio y Vespasiano- como de una colonia romana, le parecía «intéressent» pero «difficile».

Si hasta este momento toda la investigación europea de más alto nivel en temas provinciales se había ido implicando en esta cuestión -alemana, francesa, italiana- en los años ochenta también asistimos a la irrupción de la anglosajona. En un estudio de N. K. Mackie (1983: 349) sobre las colonias augústeas en el norte de África, la investigadora opinaba, con relación a este tema, que una contributio entre ciudades tan alejadas como Ilici de Icosium planteaba serios problemas de gestión, no comparables a los casos que se conocían de administraciones alejadas, como era el de las Baleares con el conventus iuridicus de Carthago Nova, menos problemático al no ocuparse de cuestiones que afectaban a la vida cotidiana. Por el contrario, para justificar la contributio icositana, habría que buscar un importante motivo, y como no se conoce ningún vínculo anterior entre las dos ciudades, N. K. Mackie (1983: 350) proponía que el texto pliniano nos presentara un traslado enmascarado de población a Hispania, similar al de Zulil y Tingi, motivado por la necesidad de dejar espacio en Icosium para un asentamiento de colonos procedentes de la cercana Rusguniae. En realidad, para esta investigadora, Plinio no se referiría a un acontecimiento que afectara a todos los icositani sino a «some Icositani», esto es, sólo una parte de la población icositana habría sido trasladada a Ilici. Esta contributio revestía un «non technical sense», pues enmascaraba un movimiento parcial de población, poco relevante para la ciudad africana, pero un «interesting fact» en la historia de Ilici.

Así las cosas, a principios del decenio siguiente, se publicó un importante corpus numismático de las acunaciones provinciales romanas, a cargo para Hispania de P. P. Ripollés (Burnett et al., 1992: 97-99), que en lo referente a Ilici reviste un gran valor al revisar todas las amonedaciones romanas de la ciudad. Especialmente interesante para nuestro tema es la última de las emisiones atestiguadas, la quinta, de época de Tiberio, donde en los reversos de sus ases aparecen dos figuras de togados dándose las manos, que si bien para P. P. Ripollés (Burnett et al., 1992, nº 198-199: 98-99) están en relación con personajes como Druso y Germánico, Tiberio y Sejano, Nerón y Druso o Tiberio y Calígula, también recoge la sugerencia de S. Price de que pudieran ser la representación de la contributio entre Ilici e Icosium. Una prueba de hasta qué punto el debate había rebasado el campo histórico para alcanzar, incluso, el numismático.

Hemos de esperar a principios de la última década del siglo XX para contar con la primera aportación española a la cuestión, en un trabajo de E. Ortíz de Urbina y J. Santos (1994: 1106), enmarcado en la incidencia del derecho latino sobre las ciudades norteafricanas, en el que se hacía hincapié en que la obtención del ius Latii por Icosium fue el resultado de un proceso de evolución histórica y de asimilación, que 
habría arrancado de la presencia inicial de agrupaciones de cives Romani. Al analizar el caso de Icosium y constatar que nos hallamos ante la única evidencia de una colonia de derecho latino en el Norte de África -y en el que asumían que la contributio estaría viva entre el 33-25 a.C. y la constitución de Mauritania como provincia romana-, los autores se hacían eco de las opiniones anteriores y consideraban que los icositani de Plinio «serían la agrupación de ciudadanos romanos - no todos los habitantes de la comunidad- que estaban unidos directamente a Ilici, si consideramos que la contributio se establecía entre comunidades cuyos ciudadanos tenían el mismo estatuto personal. Esta agrupación de ciudadanos propiciaría la asimilación progresiva de los modelos políticos y culturales romanos por el conjunto de la comunidad y explicaría la simultaneidad entre concesión de derecho latino y el estatuto colonial» (Ortíz de Urbina y Santos, 1994, 1101).

Para finalizar este primer bloque de propuestas historiográficas, que coincide con las postrimerías de la centuria, debemos reseñar la aportación directa al tema que realiza M. Coltelloni-Trannoy (1997: 130, n. 76), dentro del marco general de su estudio sobre los reinados de Juba II y Ptolomeo. Para la investigadora francesa, la contributio de Ilici con Icosium, que presentaba ventajas económicas, pues permitiría ahorrar en gastos administrativos, fue una herencia del pasado cultural de ambas ciudades, oficializando y romanizando lo que era una antigua tradición púnica. No compartía la hipótesis de N. K. Mackie de que, más que un lazo jurídico y administrativo, fuera una transferencia de población como la de Zulil y Tingi, ni tampoco que sus gentes participaran en la constitución de la colonia de Rusgunae. Consideraba que cabían dos hipótesis diferentes para explicar la contributio. Por un lado, que existiera un conventus civium Romanorum en Icosium, y que, por tanto, los icositani sólo fueran «certains habitants d'Icosium», entre los que habría ciudadanos romanos y africanos con la ciudadanía. En este contexto, los dunviros de Ilici tendrían la jurisdicción menor y el gobernador de la provincia Tarraconense la mayor. Este supuesto crearía, según M. Coltelloni-Trannoy, una «complexité administrative» que se vería agravada por la distancia. La inscripción de un ciudadano de origen indígena y magistrado de Icosium expresando su agradecimiento a Ptolomeo, sería una prueba de que los ciudadanos romanos eran ajenos a la autoridad del rey. Pero, según la investigadora, la existencia de magistraturas se conciliaría mal con un conventus. La otra posibilidad era que Icosium se hubiera transformado en un municipio, como pensaba J. Desanges, escapando a la tutela real (Coltelloni-Trannoy, 1997: 131 y n. 82), algo que beneficiaría a Roma, al contar con un enclave suplementario para su poder, sancionar de este modo la romanidad de los ciudadanos y servir de base al futuro título colonial. Sería una forma de dar coherencia administrativa respetando la tradición púnica, aunque fuera al precio de «certaines aberrations géographiques». M. ColtelloniTrannoy (1997: 131-132) se mostraba partidaria de que las colonias mauritanas, o al menos una parte de ellas, hubieran pasado a depender de la Bética y que la contributio entre Icosium e Ilici fuera un indicador de relaciones previas ibero-mauritanas, que Augusto integraría en su cuadro administrativo norteafricano.

\section{LA POLÉMICA REDIFINIDA}

Llegados a este punto, el panorama de la investigación dio un vuelco en 1996, a raíz de la aparición de una pequeña placa de bronce durante una campaña de excavación en la Alcudia de Elche. ${ }^{5}$ La pieza en cuestión recoge el reparto (sortitio) de 130 yugadas entre diez personas, tres de las cuales proceden de Icosi (sic). Aunque los primeros publicistas de la inscripción se limitaban a insinuar una posible relación entre este topónimo y los icositanos mencionados por Plinio (Chao et al., 1999: 422), en el extenso estudio que le dedicó poco después de su aparición J. Corell $\left(1999, \mathrm{n}^{\circ}\right.$ 12: 63-67; 2012, no 12: 45-49, n. 56) no se albergaban dudas sobre este hecho y, además, se la identificaba, siguiendo la tradición historiográfica, con la localidad norteafricana de Argel, mientras se añadía la propuesta de que los tres icositanos, junto a los restantes individuos consignados en la sortitio, pudieran ser descendientes de colonos itálicos.

Muy poco después, A. Faria (1999: 34-35), en un prolijo repaso de las principales novedades arqueológicas peninsulares, ofrecía nuevos puntos de vista en torno a esta cuestión. Así, el investigador portugués rechazaba la opinión de J. Corell, inclinándose por que sí se tratara de veteranos y no de civiles. La cuestión de la contributio podría tener un reflejo las abreviaturas C.C.I.A de las amonedaciones de la colonia ilicitana, que permitían ofrecer para la segunda letra,

\footnotetext{
5. Chao et al., 1999, 418; Corell J., $1999=2012, \mathrm{n}^{\circ} 12$; HEp 9, 1999, $27=A E$ 1999, $960=2001, \mathrm{n}^{\circ} 01251 ;$ HEp 14, 2005, 13 = Olesti - Molina, 2007, 1045: Sicci iug(era) CXXX (centum triginta), et traiec(tus) / ex l(imite decumano) V (quinto), (cardine) III (tertio), iug(era) VI (sex) s(emis) et ex / l(imite decumano) IIII (quarto), (cardine) III (tertio), iug(era) VI (sex) s(emis) h(ominibus) X (decem) / superist iug(era) XI (undecim) in sin(gulos) iug(era) XIII (tredecim). / C(aius) Annius C(aii)f(ilius) Gal(eria tribu) Seneca /Icos(i) / C(aius) Aufustius C(aii) f(ilius) Gal(eria tribu) Icosi / C(aius) Tettius C(aii) f(ilius) Sca(ptia tribu) Praeneste / M(arcus) Marius M(arci) f(ilius) Gal(eria tribu) Vibone / L(ucius) Aemilius L(ucii) f(ilius) Hor(atia tribu) Ulia / P(ublius) Horatius P(ublii) f(ilius) Quir(ina tribu) Malaca / C(aius) Marius C(aii) f(ilius) Vet(uria tribu) Corduba / L(ucius) Valerius L(uci) f(ilius) Fal(erna tribu) / Aurelia Cariss(a) / L(ucius) Fabius L(ucii) f(ilius) Gal(eria tribu) / Icosi / Q(uintus) Fufius Q(uinti) f(ilius) Mae(cia tribu) / Baliaricus. Hemos mantenido la lectura de J. Corell con excepción de la 1. 4, en la que la mayoría de los publicistas se inclinan, con razonamientos que compartimos, por la que recogemos.
} 
entre otras posibles reconstrucciones, la de la palabra C(ontributa).

$\mathrm{Si}$, como cabía esperar, hasta este momento la pieza había generado un gran impacto en el campo epigráfico y arqueológico, iba a ser en el ámbito de los estudios agronómicos romanos donde, como era de esperar, a partir de ahora alcanzaría el mayor protagonismo. Fue en este contexto en el que, tangencialmente, M. Mayer y O. Olesti (2001: 113-115) sugirieron, como más probable, que la ciudad de Icosium se pudiera encontrar en las proximidades de Elche, habida cuenta que la cita pliniana describía su ora maritima, si bien, no llegaban a descartar por completo la tradicional localización africana de los icositani. Por otra parte, estos investigadores se sumaban a la opinión de que los individuos de la sortitio eran veteranos, proponiendo incluso que, al ser ciudadanos, estos pobladores fueran el resultado del reclutamiento de la conocida legio Vernacula, levada por Varrón, y asentados en Ilici en época triunviral o, mejor, augústea. Según los cálculos de los autores, se habrían ubicado 900 veteranos, el equivalente a 1,5 cohortes, a los que habría que sumar sus familias.

A este trabajo, fundamentalmente agronómico, siguieron de inmediato otros, como los de E. Ariño et al. (2002) y los comentarios de R. González Villaescusa (2002), que incidían sólo en cuestiones técnicas sin referencias a la cuestión contributense, pero importantes porque sirvieron de antecedente al estudio de Y. Guillaumin (2002: 116, n. 9), que, de forma explícita, apoyaba la localización de Icosium en la península ibérica. Casi simultáneamente, O. Olesti y J. Molina (2002: 1050-1053), en un trabajo en el que abordaban de nuevo diversas reflexiones sobre el catastro ilicitano, mantenían las mismas propuestas sobre el número $\mathrm{y}$ origen de los veteranos que ya fueron defendidas, según acabamos de ver, por uno de sus autores.

Algo anterior a estas publicaciones, y sin aún tener en cuenta la sortitio de Ilici, debemos mencionar una aportación complementaria de E. Ortiz de Urbina (2000: 42-43, n. 61 y 115-116), en una monografía sobre el Ius Latii en Hispania. Como refuerzo a la tesis de J. Desanges de la fundación de una colonia romana -y no latina- en Icosium por Vespasiano, subrayaba el hecho de que apareciera en la mencionada inscripción del CIL VIII 20853, una vez implantado el régimen colonial, un duumvir quinquennalis, magistratura suprema que, recordaba esta autora, por el momento sólo se hallaba reflejada en colonias o municipios romanos. A menos que, como también pensaba J. Desanges, la inscripción procediera de Rusguniae, E. Ortiz de Urbina recopilaba una serie de testimonios epigráficos que, en su opinión, confirmarían la posibilidad de que en época flavia Icosium se transformara en colonia romana.

Sin lugar a dudas, la aportación más determinante del nuevo siglo vino de la mano de G. Alföldy. Inserto en un estudio global de las ciudades de la actual provincia de Alicante, en relación a la compleja y debatida fecha de la fundación de la colonia de Ilici, el investigador húngaro realizaba importantes propuestas sobre los primeros años de la colonia ilicitana. Se mostraba partidario de fijar una primera fundación en época de Lépido, en el año 42 a.C. y, siguiendo tanto criterios numismáticos como epigráficos, una segunda en época augústea (G. Alföldy, 2003: 6- 8 y 12-13). Defendía esta última en base a dos testimonios concretos. En primer lugar, la inscripción de T. Statilius Taurus, procónsul de Hispania Citerior entre el 29/28 a.C., en la que aparece como patronus de la colonia y, por tanto, posible deductor de la misma hacia el año 26 a.C. (Alföldy, 2003: 9). ${ }^{6}$ En segundo lugar, la sortitio, en la que los tres individuos icositanos procederían, en su opinión, del conventus civium Romanorum constituido entre el 33 y el 25 a.C., algo que permitiría datar la inscripción de Ilici en el último tercio del siglo I a.C. (Alföldy, 2003: 10). Los apelativos de Iulia y Augusta de sus monedas vendrían a ser la evidencia de las dos fundaciones. Además, para G. Alföldy (2003: 11 y 13), ambas deductiones habrían dejado su huella en dos significativos privilegios de la colonia, el de la inmunitas, del que nos informa Plinio en III 4, 19, que llegaría con la primera fundación, y el ius Italicum, del que nos da cuenta el Digesto, y que entraría en vigor con la deductio de Augusto. ${ }^{7}$ Para G. Alföldy (2003: 12-13) estaba fuera de dudas que nos encontrábamos ante una colonia de veteranos, no sólo porque las monedas presentan los símbolos militares, sino también por el testimonio de la sortitio. Los diez ciudadanos que allí aparecen llevaban tribus antiguas, pues según los cálculos de G. Alföldy, recibieron la ciudadanía antes que sus comunidades, ya que habrían nacido entre el 65 y el 55 a.C. y habrían obtenido las tierras tras veinte años de servicio, en el año 26 a.C. o poco más tarde, cuando Estatilio Tauro fue honrado como patrono y la ciudad ya ostentaba el apelativo de Augusta. En cuanto a los icositani, pensaba G. Alföldy (2003: 13), presentaban una situación diferente. Podría tratarse de antiguos soldados o negociantes asentados primero en el conventus civium Romanorum de Icosium, que pasarían después a ser asociados a la colonia ilicitana por medio de un praefectus, e inscritos en la tribu de la ciudad cuando se realizó la segunda fundación augústea. Los planteamientos de G. Alföldy encontraron una inmediata aceptación en la historiografía española, siendo plenamente asumidos por J. M. Abascal (2004: 81-82 y 86).

A partir de este momento asistimos en el mundo de la investigación a una etapa de nuevas revisiones y reformulaciones. En ellas se inscribe, en primer lugar, la aportación de J. Gascou (2004-2005: 262), que

6. CIL II 3556 = Rabanal - Abascal, 1985, no 64, 224-225; Corell, 1999, $\mathrm{n}^{\circ} 4,54-56=2012, \mathrm{n}^{\circ} 4,36-39:$ (ito) Statilio $/$ Tauro imp(eratori) / III co(n)s(uli)/ II / patrono.

7. Dig. L 15, 8: In Lusitania Pacenses et Emeritenses iuria Italici sunt. Valentini et Licitani habent... 
proponía ahora una serie de fases por las que discurriría Icosium, partiendo de la base de que una ciudad peregrina no estaba en condiciones de establecer una contributio con una colonia y, como creían L. Teutsch y U. Laffi, sólo afectaría a los habitantes romanos de la comunidad africana, organizados en un conventus civium Romanorum, a los que Plinio habría agrupado bajo el término genérico de Icositani. Según J. Gascou, en la primera fase de Icosium, entre el 33 y el 25 a.C., asistiríamos a la instalación de veteranos por Octavio, organizados en un convento en el seno de la ciudad peregrina, que fue ligado administrativamente a Ilici a partir del 25 a.C., para evitar que los ciudadanos romanos quedaran bajo la autoridad del nuevo rey mauritano y gozaran, por el contrario, de un estatuto de extraterritorialidad. Desde ese momento, la ciudad peregrina quedó bajo la potestad de los reyes mauritanos, mientras que, por el contrario, el convento dependió de Ilici. Algunos peregrinos icositanos recibieron la ciudadanía, como podría ser el caso de Caecilius Rufus, que habría ejercido las magistraturas en la ciudad peregrina, y que no habría alcanzado este privilegio por formar parte de la colonia de Ilici, sino porque, él o su padre, la habrían obtenido a título individual. Con la anexión de Mauritania como provincia en el 40 d.C., el convento fue incorporado a la ciudad indígena de Icosium. J. Gascou (2004-2005: 262-263) no compartía la opción de J. Desanges de que Icosium, antes de transformarse en colonia romana con Vespasiano, hubiera podido ser municipio, al no existir prueba alguna, ni resultar necesario. Por el contrario, estimaba que era más prudente pensar en una única transformación colonial en época flavia, a datar entre el 74-76 d.C., como mostraba el texto de Plinio y la inscripción romana de su primer pontífice. En cambio, admitía ahora la propuesta de lectura del texto de Plinio V 20 de J. Desanges, que establecía una comparación entre lo que serían dos colonias romanas honorarias, Caesarea e Icosium, pues así desaparecía la anómala presencia de una colonia latina en África del norte en tiempos de Vespasiano. Entonces se produciría la fusión de los habitantes romanos descendientes de los veteranos romanos, organizados en un conventus, con los habitantes peregrinos, de ahora en adelante transformados en ciudadanos de una colonia romana. En suma, para J. Gascou (2004-2005: 263-264), Icosium habría contado primero, entre el 33-25 a.C., con un conventus $c$. $R$.; a partir del 25 a.C. el conventus establecería la contributio con Ilici, mientras la ciudad peregrina dependería de los reyes mauritanos, aunque algunos de sus habitantes alcanzaron la ciudadanía; en el 40 d.C. el conventus dejaría de depender de Ilici y pasaría, como ente autónomo, a vincularse a la ciudad peregrina de Icosium; por último, a comienzos del periodo flavio, ambas comunidades se integrarían en una única colonia honoraria de ciudadanos romanos.

En este proceso revisor, A. Faria (2006: 220-224) también retomaba la cuestión, ya planteada en su anterior artículo. Se mostraba ahora partidario de rebajar la cronología de la fundación de la colonia, sobre todo por la datación de la sortitio, que consideraba posterior al 25 a.C. La presencia de ciudadanos icositanos de la tribu Galeria y el patrocinio de Statilus Taurus suponían, para A. Faria, evidencias de mucho peso para inclinarse por una primera fundación entre el 29 -28 a.C., datando el comienzo de las series numismáticas a partir del 25 a.C. Oficialmente, la ciudad se denominaría colonia Caesarea Ilici, colonia Caelestis Ilici o colonia Concordia Ilici; más tarde, después del 27 a.C. o, seguramente, entre el 15-12 a.C., se añadiría el epíteto Augusta. Tampoco descartaba que entonces pasara a llamarse colonia Contributa Ilici Augusta o colonia Coniuncta Ilici Augusta. Finalmente, a partir del 2 a.C., transitaría a colonia Iulia Ilici Augusta, colonia Inmunis Ilici Augusta o colonia Iunionia Ilici Augusta. Admitía como viable la posibilidad de que la contributio se reflejara en las monedas.

En este marco, se presentó, poco después, una valiosa aportación por O. Olesti y X. Espluga (2007). Destacaban la gran precisión del documento de $a d$ signatio de tierras evidenciada en la sortitio ilicitana, realizada en una colonia inmunis y con ius Italicum, y que se manifestaba en el cuidado por preservar las tierras que se mantenían en manos públicas (subseciva). El pequeño tamaño de los lotes asignados por colono (3 ha), se vería compensado por las exenciones fiscales de la colonia. Para los autores, los colonos de la sortitio eran, muy probablemente, veteranos, similares a los que figuran en otras asignaciones militares que recogen los textos de colonias hispanas (v. g. Emerita), y confirman los símbolos militares de las monedas de Ilici, aunque no es posible precisar si procedían todos de una misma legión o de varias. Aceptaban estos autores la propuesta de G. Alföldy de una doble deductio, en el 41 y a partir del 29 a.C., pero tampoco descartaban una única en esta última fecha. También siguen al mismo investigador en lo que hace referencia a Statilius Taurus, patrono a partir del 26 a.C., al que ven como deductor de la colonia, y del que recuerdan sus dos triunfos, uno sobre África, en el 34 a.C., y otro posterior, en el 29 a.C., por su participación en las guerras cántabras. La fundación del Portus Ilicitanus por Augusto es, según ellos, clave para el desarrollo de la colonia. En cuanto al origen de los colonos, los autores reconocen que nos hallamos ante una cuestión difícil. Destacan que Casius Longinus en el 48 a.C. reclutó hispanos en ciudades de la Bética, la llegada de tropas de África e, incluso, la presencia de fuerzas africanas del rey Bogud, circunstancias que estarían en la base del asentamiento de veteranos en Ilici, recogiendo así la opinión de G. Alföldy de que se trataría de hispanos, levados tanto en esas ciudades béticas como, también, en ciudades italianas. En cuanto a lo que llaman «the Icosium affair», consideran, como G. Alföldy, que los tres icositani no eran veteranos sino ciudadanos trasladados desde Icosium a Ilici. Las razones, según ellos, serían dos. De un lado, que las ciudades de Mauritania oriental estaban unidas a la Bética, y que algunas de 
Hispania jugaran en ello un papel destacado, como $\mathrm{Ili}$ ci, Carthago Nova, Gadir o Iulia Traducta. Los icositanos de Ilici podrían ser hijos de ciudadanos romanos establecidos en el conventus de Icosium antes del 33 a.C., reclutados por el ejército, y licenciados en el 29 a.C. A diferencia de lo que opina G. Alföldy, la tribu Galeria que ostentan podría no estar conectada con $\mathrm{Ili}$ $c i$ sino haberla adquirido con anterioridad. Creen que la contributio fue posterior a la fundación de Ilici y que la presencia de los icositanos en la deductio - una consecuencia de la entronización de Juba II en el reino mauritano el 25 a.C.-, conduciría a la contributio. Pero si, por otro lado, se considera la deductio contemporánea a la contributio, a partir del 25 a.C., ya no sería necesario ver como veteranos a los icositanos de la sortitio, pues en aquel momento ya serían ciudadanos y, por tanto, tendrían la tribu de la ciudad. Finalmente, los autores recuerdan como la Lex Ursonensis en su cap. 103. 3 habla de col(onos) incolasque contributos quocumquo tempore colon(iae), lo que puede interpretarse como que en las fundaciones coloniales se empleara contributenses. Los 270 icositanos que pudieron participar en la sortitio, habrían sido el monto total de aquellos que formaban parte del conventus civium Romanorum de Icosium.

La publicación de un corpus de la epigrafía latina republicana de Hispania por B. Díaz (2008: 87-88) supuso una nueva aportación al tema. Coincide este autor en que los colonos de la sortitio formaban parte de antiguas familias de inmigrantes instaladas en ciudades aún peregrinas. En cuanto a los tres colonos icositanos, aunque recoge las opiniones que defienden su procedencia de la ciudad del norte de África, ve como más razonable la propuesta de $\mathrm{M}$. Mayer y O. Olesti. Considera el término Icosi como indeclinable lo que avalaría que se trate de una ciudad indígena española. La presencia de tres de sus naturales en la deductio podría deberse, o bien a una expropiación de tierras en beneficio de la nueva fundación, o bien a la participación de un número importante de icositani. B. Díaz defiende una ascendencia itálica para estas familias y considera que el que ostenten la Galeria es consecuencia de su acceso a la ciudadanía tras la participación en la fundación de Ilici, pues debió ser esta la tribu que se asignó a la ciudad, aunque no pueda demostrarse. Finalmente, se inclina por una datación de la sortitio durante el mandato de Lépido, aunque atestigua la otra opción augústea (Díaz, 2008: 88-89, n. 14 y 15).

Un quinquenio más tarde, aparecen dos estudios que vuelven a ocuparse de nuestro tema. De un lado, el de L. Amela (2013: 133-134), que, en relación con los icositanos, se limitaba a sumarse a las propuestas del citado corpus. Más amplia es, por otra parte, la atención que le presta C. Falomir (2013: 132-135 y 137), en el seno de una tesis sobre el reino de Juba II. Tras una recopilación de las opiniones más importantes, desde el punto de vista de la investigadora, le parece aceptable que las inscripciones que hablan de los cargos de Caecilius Rufus correspondan a Rusguniae y que, por tanto, no deben ser tomadas en consideración para este caso. C. Falomir (2013: 136-137) opina que Icosium albergaría, quizás, un conventus $c$. R., apoyando así la teoría de N. K. Mackie de que algunos icositanos fueron trasladados a Ilici, de lo que sería demostrativa la sortitio, aunque no le resulte claro si eran ciudadanos romanos antes de su llegada a la ciudad hispana. Considera que el resto de Icosium fue, durante todo el tiempo, una ciudad peregrina, que como tal, no fue contributense de Ilici, sino dependiente de los reyes mauritanos.

\section{UN DEBATE ABIERTO}

Dentro de la fase actual de replanteamientos de posturas previas, M. Coltelloni-Trannoy también ha llevado a cabo recientemente una reciente revisión de sus opiniones sobre el tema. En un trabajo centrado exclusivamente en el origen de los icositani, y tras un repaso general de la evolución del norte de África en época augústea, la investigadora analiza la contributio desde dos aspectos. Por una parte, el que deriva de la inscripción de L. Caecilius Rufus, al que considera un magistrado de Icosium, hijo de un peregrino, unido a su patria y al rey de Mauritania por lazos afectivos, elementos que demostrarían que la autonomía de la ciudad no se habría visto mermada por la contributio. (Coltelloni-Trannoy, 2012: 142-143). Puesto que la hipótesis de que fuera una ciudad peregrina le parece inconcebible para una ciudad unida a una colonia y en un territorio no provincial, ni tampoco le resulta convincente la propuesta del conventus civium Romanorum, se muestra partidaria de renunciar a la contributio entre la ciudad africana y la hispana, y, haciéndose eco de la opinión de M. Mayer y O. Olesti, propone una Icosi española y una Icosim/Icosium africana, pues las coincidencias históricas y lingüísticas púnicas entre el norte de África y la península ibérica le parecen suficientes para avalar esta posibilidad (Coltelloni-Trannoy, 2012: 142-146). Los argumentos concretos que aduce son tres: que el cognomen de uno de los icositanos receptores de tierras de la sortitio, C. Annius Seneca, es muy frecuente en Hispania; que hay una concomitancia de las tribus de los tres colonizadores icositanos, la Galeria, con la de Ilici, lo que le sugiere que ya formaban parte de su cuerpo cívico; y, por último, que, si aún no se ha localizado un emplazamiento en Hispania que responda a estas características, es porque se trataba de una comunidad pequeña que habría acabado siendo absorbida en época imperial y de la que, por ello, no nos ha quedado rastro, pero que algún día puede ser descubierta en la vecindad de Ilici, acomodada a la traducción púnica de Icosim («isla de los búhos» o «isla de las gaviotas»), esto es, en un lugar que resulte favorable al fondeo y atraque de barcos (Coltelloni-Trannoy, 2012: 146-147).

El análisis de las relaciones contributenses norteafricanas, tanto de Cirta con Mileu, Rusicade y Chullu, 
por un lado, y de Ilici con Icosium, por el otro, ha sido el objetivo del trabajo que, muy poco después, ha publicado G. Bernard $(2014,72)$, en el que, en cuanto al tema que tratamos, plantea tres cuestiones: el origen de los icositani, la naturaleza de las relaciones entre Ilici e Icosium y su estatuto jurídico. A partir del texto de la sortitio, la investigadora da como válida la existencia de dos fundaciones coloniales, una con Lépido y otra, entre el 26 y 19 a.C., con veteranos de las guerras civiles, donde se destaca la presencia de tres icositani como beneficiarios de la entrega de tierras en el reparto de Ilici (Bernard, 2014: 73-74). Con relación al origen de estos icositani, recuerda cómo hasta la publicación de la sortitio todos los autores defendían su procedencia de Icosium, la actual Argel, pero también que, a partir de M. Mayer y O. Olesti, ha habido una propuesta alternativa de que fueran originarios de la zona ilicitana. Para G. Bernard (2014: 75-76), no hay rastro de la presencia del topónimo Icosi en península ibérica, y, por otra parte, le parece justificable que Plinio, que copia la información de la formula provinciae de Augusto, haga referencias paralelas a uno y otro continente, por lo que le resulta difícil admitir que haya existido un Icosi español alternativo. Que en un periodo posterior a la creación del reino de Mauritania una ciudad del norte de África como Icosium haya pasado a la dependencia de Ilici se explica bien en este contexto. Según G. Bernard, no hay ningún problema en que los ciudadanos romanos de Icosium quedaran subordinados a la ciudad hispana y, por tanto, a la provincia Tarraconense y que, incluso, hasta que hubiera habido un traslado de población, como había ocurrido con Zulil y Tingis. Para esta investigadora, nada impide la relación entre ambas ciudades a ambas orillas del Mediterráneo y, por tanto, no resulta necesaria la existencia de una comunidad en Hispania del mismo nombre que la africana, pues, aunque resulte más lógica geográficamente, no se explica ni en el plano epistemológico ni en el histórico. En cuanto al estatuto de Icosium tras el cese de la contributio, G. Bernard (2014: 78-79) comparte la propuesta de J. Desanges y considera que obtuvo el derecho colonial romano, no el latino, con Vespasiano, de forma que antes habría sido un municipio romano, un oppidum o un pagus, similares a los de Uchi Maius o Thugga. Participa de la opinión de G. Alföldy de que los veteranos romanos de Icosium se incorporaron como comunidad contributense a la administración de Ilici. La insignificancia de la ciudad le habría impedido, como en otros casos, llegar a ser colonia autónoma, pero habría conservado sus propias magistraturas y sacerdocios, aunque bajo la supervisión de un praefectus. G. Bernard (2014: 7980) piensa que la adopción de este modelo de dependencia por Ilici se basaría en la existencia de relaciones antiguas, de época ibérica o púnica, así como -al igual que opina G. Alföldy- en el atractivo de la inmunitas ilicitana. La contributio finalizaría en el 42 d.C.

Como última aportación al tema hemos de referirnos a la que realiza, nuevamente, O. Olesti, (2014:
225-226, n. 4) que, tras un repaso de la situación anterior a la fundación colonial, considera que la misma se produjo en época triunviral, aunque aplicando una iniciativa anterior de César. Reitera la posibilidad, también, de una segunda fundación augústea, tanto por las monedas como por el patrocinio de T. Statilius Taurus. En cuanto a la contributio, rechaza la opinión de G. Alfoldy de que la sortitio fuera realizada entre el 33-27 a.C., e insiste en situarla en época triunviral, pues no considera concluyente que la presencia en el documento de tres icositani obligue a establecer fechas bajas. Defiende, de nuevo, que el Icosium del bronce ilicitano podría no ser el africano sino un núcleo hispano indígena, como habría expuesto, con motivos lingüísticos, B. Díaz, y propone también que estuviera en el pueblo de Agost, donde señala la existencia de una necrópolis, indicio de un núcleo urbano importante (Olesti, 2014: 226, n. 5). Pese a todo, en último extremo, no llega a descartar por completo una ubicación africana de los icositani (Olesti, 2014: 244).

Hasta aquí el debate. Como hemos podido comprobar, no exagerábamos al calificar de un tema candente la controversia historiográfica sobre la contributio entre Ilici e Icosium, marcada por una discusión que, con el paso de los años, ha ido dilatando su amplitud y profundizando en complejidad, enfrentada por discrepancias en cuestiones clave: la cronología precisa de su constitución y cese; la existencia o no de un conventus civium Romanorum; las causas de la creación del vínculo contributense; el estatuto de Icosium en el momento de la disolución de la ligadura; y, asunto determinante, la localización de los icositani de Plinio en Hispania o en el norte de África. Ponerlas sobre la mesa es, no se le escapará al lector, además de un esfuerzo recopilador, una invitación a seguir buscando respuestas esclarecedoras a problemas que distan, hoy por hoy, de estar resueltos. Nihil desperandum.

\section{REFERENCIAS}

Abascal, J. M. (2004). Colonia Iulia Ilici Augusta. En L. Abad y M. Hernández (Eds.). Iberia, Hispania, Spania. Una mirada desde Ilici (pp. 79-94). Alicante: Caja de Ahorros del Mediterráneo.

Albertini, E. (1923). Les divisions administratives de l'Espagne romaine. Paris: De Boccard.

Alföldy, G. (2003). Administración, urbanización, instituciones, vida pública y orden social. Canelobre, 48, 35-57.

Amela, L. (2013). Sobre el origen de la Colonia C. Ilici Augusta. Una nota. Arse, 47, 125-138.

Ariño, E., Gurt, J. M., Palet, J. M. (2002). La inscripción catastral de Ilici. Ensayo de interpretación. Pyrenae, 31-32, 223-226.

Bernard, G. (2014). Le lien de contributio entre colonies et communautés de citoyens romains dans les provinces africaines. Réflexions sur les colonies de la Confédération 
cirtéene et sur la contributio entre Icosium et Ilici. En C. Briand-Ponsard (Ed.). Centres de pouvoir et organisation de l'espace, Actes du X $X^{e}$ colloque international sur l'histoire et l'archéologie de l'Afrique du Nord préhistorique, antique et médiévale (Caen, 25-28 mai 2009) (pp. 65-80). Caen: Presses universitaires.

Blázquez, J. Ma . (1978). Relaciones entre Hispania y África desde los tiempos de Alejandro Magno hasta la llegada de los árabes. Economía de la Hispania romana (pp. 647-670). Bilbao: Nájera Ediciones.

Burnett, A., Amandry, M., Ripollés, P. P. (1992). Roman Provincial Coinage (RPC), I: From the Death of Caesar to the Death of Vitellius (44 BC-AD 69). London-Paris: British Museum Press.

Chao, J. J., Mesa, J. F., Serrano, M. (1999). Un nuevo bronce en la Alcudia. En J. González (Ed.). Ciudades privilegiadas en el Occidente romano (pp. 417-424). Sevilla: Secretariado de Publicaciones de la Universidad de Sevilla.

Coltelloni-Trannoy, M. (1997). Le royaume de Maurétanie sous Juba II et Ptolémée (25 av. J.-C. - 40 ap. J.-C.). Aixen-Provence: CNRS Editions.

Coltelloni-Trannoy, M. (2012). Encore les Icositani.... En B. Cabouret, A. Groslambert y C. Wolff (Eds.). Visions de l'Occident romain. Hommages à Yann Le Bohec, I (pp. 137152). Lyon-Paris: Etudes et Recherches sur l'Occident Romain. CEROR. De Boccard.

Corell, J. (1999). Inscripcions romanes d'Ilici, Lucentum, Allon, Dianium $i$ els seus territoris (IRILADT). Valencia: Nau Llibres

Corell, J. (2012). Inscripcions romanes del País Valencià VI. Ilici, Lucentum, Allon, Dianium i els seus territoris (IRI$L A D T)$. València: Universitat de València.

Cortés y López, M. (1836). Diccionario geográfico-histórico de la España antigua, Tarraconense, Bética y Lusitana, con la correspondencia de sus regiones, ciudades, montes, ríos, caminos, puertos e islas a las conocidas en nuestros dias. Tomo III. Madrid: Imprenta Real.

Desanges, J. (1980). Pline l'Ancien. Histoire Naturelle. Livre V, ler partie. 1-46. L'Afrique du Nord. Paris: Les Belles Letres.

Díaz, B. (2008). Epigrafía latina republicana de Hispania. Barcelona: Colección Instrumenta, Universitat de Barcelona.

Falomir, C. (2013). Juba II, rey de los mauros y los libios. (Tesis Doctoral). Universitat de València. Recuperado de: http://roderic.uv.es/handle/10550/28428.

Faria Marques de, A. (1999). Colonização e municipalização nas províncias hispano-romanas: reanálise de alguns casos polémicos. Revista portuguesa de arqueologia, 2(2) $29-50$.

Faria A. Marques de, A. (2006). Novas notas historiográficas sobre Augusta Emerita e outras ciudades hispano-romanas. Revista portuguesa de arqueologia, 9(9), 211-237.
Gascou, J. (1981). Tendences de la politique municipale de Claude en Maurétanie. Ktéma, 6, 227-238.

Gascou, J. (1982). Politique municipale en Afrique du Nord. I-II. De la mort d'Auguste au debut du III ${ }^{\text {e }}$ siècle. En H. Temporini y W. Haase (Ed.). Aufstieg und Niedergang der Römischen Welt (ANRW) II, 10.2 (pp.136-229). Berlin-New York: W. de Gruyter.

Gascou, J. (2004-2005). Sur le statut de quelques villes de Numidie et de Maurétanie Césarienne. Antiquités Africaines, 40-41, 259-267. DOI: http://doi.org/10.3406/ antaf.2004.1391

González Villaescusa, R. (2002). Las formas de los paisajes mediterráneos. Ensayos sobre las formas, funciones y epistemología parcelarias. Estudios comparativos en medios mediterráneos entre la Antigüedad y la época moderna. Jaén: Universidad de Jaén.

Gsell, S. (1921-1928). Histoire ancienne de l'Afrique du Nord, VIII. Osnabruck. Otto Zeller.

Guillaumin, J.-Y. (2002). Note Sur le document cadastral romain découvert à La Alcudia (Elche, Province d'Alicante). Dialogues d'histoire ancienne, 28(1), 113-134. DOI: http:/ doi.org/10.3406/dha.2002.2463

Ibarra, A. (1879). Illici, su situación y antigüedades. Alicante. Instituto de Estudios Alicantinos.

Keune, J. B. (1918). Icositani. Realencyclopädie Altertumswischenchaft (RE). Suppl. III (col. 1195). München: Alfred Druckenmüller Verlag.

Kornemann, E. (1940). Contributio. Realenciclopädie Altertumswischenchaft (RE). Suppl VII. (cols. 91-96). München: Alfred Druckenmüller Verlag.

Laffi, U. (1966). Adtributio e contributio. Problemi del sistema politico-amministrativo dello stato romano. Pisa: Nistri Lischi Editori.

Lassère, J. M. (1977). Vbique populus. Peuplement et mouvements de population dans l'Afrique romaine de la chute de Carthage à la fin de la dynastie des Sévères (146 a.C. -235 p.C.). Paris: Éditions du Centre National de la Recherche Scientifique.

Le Glay, M. (1968). A la recherche d'Icosium. Antiquités Africaines, 2, 7-54. DOI: http://doi.org/10.3406/ antaf.1968.888

Llobregat, E. (1972a). Contestania ibérica. Alicante: Instituto de Estudios Alicantinos.

Llobregat, E. (1972b). Icositani. En M. Mas (Ed.). Gran Enciclopedia de la Región Valenciana, V. Valencia: Heraclio Fournier.

Llobregat, E. 1980. El Alto Imperio (siglos I a III). En Más Ivars (Ed.). Nuestra historia, II. Valencia: Aramo.

Mackie, N. (1983). Augustan Colonies in Mauretania. Historia, 32, 332-358.

Mayer, M. y Olesti, O. (2001). La sortitio de Ilici. Del documento epigráfico al paisaje histórico. Dialogues d'histoire 
ancienne, 27(1), 109-130. DOI: http://doi.org/10.3406/ dha.2001.2439

Olesti, O. y Espluga, X. (2007). New Perspectives about de Tabula of Ilici (Elx-Elche, Spain). En XIII Congressus Internationalis Epigraphiae Graecae et Latinae: Provinciae Imperii Romani Inscriptionibus Descriptae. Recuperado de: http://ciegl.classics.ox.ac.uk/html/webposters/60_Olesti. pdf

Olesti, O. y Molina, J. (2002). La sortitio y el catastro de Ilici. En M. Mayer, G. Baratta y A. Guzmán (Eds.). XII Congressus Internationalis Epigraphiae Graecae et Latinae: Provinciae Imperii Romani Inscriptionibus Descriptae (Barcelona, 3-8 Septembris 2002) (pp. 1045-1054). Monografies de la Secció Històrico-Arqueològica 10. Barcelona: Institut d'Estudis Catalans.

Olesti, O. (2014). Paisajes de la Hispania romana. La explotación de los territorios del Imperio. Sabadell: Dstoria Edicions.

Ortiz de Urbina, E. (2000). Las comunidades hispanas y el derecho latino. Vitoria: Universidad del País Vasco.
Ortiz de Urbina, E y Santos, J. (1994). Algunas reflexiones sobre el derecho latino en el Norte de África. L'Africa Romana, X(3), 1093-1109.

Ramos, R. (1975). La ciudad romana de Illici. Alicante: Instituto de Estudios Alicantinos.

Rabanal, A. y Abascal, J. M. (1985). Inscripciones romanas de la provincia de Alicante. Lucentum, IV, 191-244. DOI: http://dx.doi.org/10.14198/LVCENTVM1985.4.13

Teutsch, L. (1962). Das Städtewesen in Nordafrika in der Zeit von C. Gracchus bis zum Tode des Kaisers Augustus. Berlin: W. de Gruyter.

Tovar, A. (1989). Iberische Landeskunde, III. Tarraconensis. Baden-Baden: Koerner.

Vittinghoff, F. (1951). Römische Kolonisation und Bürgerrechtspolitik unter Caesar und Augustus, Wiesbaden: Franz Steiner Verlag.

Wilmanns, G. (1881). Corpus Inscriptionum Latinarum (CIL), VIII. Berolini: Academia Litterarum Regiae Borussicae. 